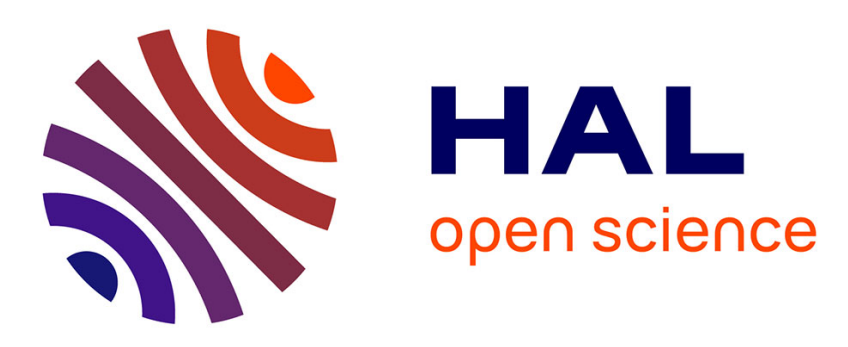

\title{
Langmuir probe in magnetized plasma: Study of the diffusion parameter
}

Jean-louis Jauberteau, Isabelle Jauberteau, Osvaldo Daniel Cortázar, Ana Megía-macías

\section{- To cite this version:}

Jean-louis Jauberteau, Isabelle Jauberteau, Osvaldo Daniel Cortázar, Ana Megía-macías. Langmuir probe in magnetized plasma: Study of the diffusion parameter. Contributions to Plasma Physics, In press, 10.1002/ctpp.202100066 . hal-03324910

\section{HAL Id: hal-03324910 https://hal.science/hal-03324910}

Submitted on 13 Oct 2021

HAL is a multi-disciplinary open access archive for the deposit and dissemination of scientific research documents, whether they are published or not. The documents may come from teaching and research institutions in France or abroad, or from public or private research centers.
L'archive ouverte pluridisciplinaire HAL, est destinée au dépôt et à la diffusion de documents scientifiques de niveau recherche, publiés ou non, émanant des établissements d'enseignement et de recherche français ou étrangers, des laboratoires publics ou privés. 


\title{
Langmuir probe in magnetized plasma: Study of the diffusion parameter.
}

J.L. Jauberteau, ${ }^{1}$ I. Jauberteau, ${ }^{1}$ O. D. Cortázar, ${ }^{2}$ A. Megía-Macías ${ }^{3,4}$.

1) Université de Limoges, UMR 7315 CNRS, IRCER, 12 rue Atlantis 87068, Limoges, France.

${ }^{2)}$ Universidad de Castilla-La Mancha, ETSII-INEI, Av. Camilo Jose Cela s/n, 13071-Ciudad Real, Spain.

3) Institute for Research in Technology, ICAI, Comillas Pontifical University, Santa Cruz de Marcenado, 26, 28015 Madrid, Spain

4) Mechanical Engineering Department, ICAI, Comillas Pontifical University, Alberto Aguilera 25, 28015 Madrid, Spain

\begin{abstract}
.
In low temperature magnetized plasma, Langmuir probe measurements must be corrected because of the electron diffusion through the sheath which is formed around the probe collector. The correction factor which is called the electron diffusion or electron sink parameter depends on many other parameters like the probe geometry, the electron diffusion coefficient, the sheath thickness or the potential profile through the sheath. Based on a previous work, we determine the values of this parameter under different experimental conditions and we study the effect of the electron energy, of the probe biased voltage and of the magnetic field intensity on this parameter. The results are compared with theoretical models published in literature. An empirical equation is determined to fit the diffusion parameter value versus magnetic field intensity.
\end{abstract}

\section{Introduction.}

The electrostatic probes are an efficient diagnostic tool to determine the plasma parameters, as the plasma potential, the electron density and the mean electron energy or the electron energy distribution function (EEDF)[1,2,3]. However, the I-V probes characteristics measured by means of electrostatics probes depend on plasma characteristics and can be drastically changed in case of high pressure or magnetized plasmas. The electron current collected by the probe at different negative bias voltages depends on the electron diffusion through the sheath produced around the collecting probe. So, the electron current measured value must be corrected to determine accurate plasma parameters. The measured current depends on a 
macroscopic parameter, which is called the diffusion parameter or the electron sink parameter. This parameter is a function of the electron diffusion coefficient, of the sheath thickness and also is correlated to the probe geometry and other parameters $[4,5,6]$. In the case of high pressure plasma, the diffusion coefficient is isotropic and depends on collision processes in the sheath. However, when a magnetic field is applied, because of the Lorentz force on charged particles anisotropy appears and the current collected by the probe changes with the effective conductivity which depends on the probe geometry, on the orientation within the magnetic field and on three main mechanisms, the ion viscosity, the ion neutral collision and the ion inertia /7/. These mechanisms are efficient particularly in fully ionized plasma and when the probe radius is larger than the ion-gyro radius. The potential in front of the probe is disturbed on a transverse scale depending on the ion-gyro radius and the potential profile through the sheath drastically changes according to the experimental conditions. The purpose of this work is to determine and to study the diffusion parameter in the case of cold magnetized plasma, corresponding to partially ionized plasma (relative ionization lower than $1 \%$ ). In this case the ion-gyro radius is larger than the probe radius (typically equal to 10 probe radius) and the conductivity within the sheath mainly depends on the electron diffusion coefficient anisotropy due to the magnetic field.

\section{Probe characteristics in the electron diffusion regime.}

In the case of high pressure plasma as well as for magnetized plasma, the electron current collected by a negatively biased probe decreases with decreasing electron diffusion through the sheath. The electron kinetic is governed by an electron diffusion regime in the sheath formed around the probe. When the region disturbed by the probe (equivalent to the sheath around the probe) is lower than the electron energy relaxation length $\lambda_{\varepsilon}$ the electron energy distribution function can be considered unchanged by the probe effect. The electron energy changes due to collisions and to the heating by the external electric field produced around the probe can be neglected [6]. The EEDF corresponding to the real EEDF within the plasma bulk is measured when the electron diffusion rate within the sheath is high enough to cope with the drain of electron from the plasma to the probe. The difference between the real EEDF and the measured EEDF increases with increasing gas pressure or radial magnetic field component, because of electron diffusion decreasing [6, 8].

In magnetized plasma the electron diffusion coefficient is anisotropic because of the electron motion in the magnetic field. In the case of a diffusion regime, assuming an isotropic EEDF in 
the plasma bulk, the electron kinetic equation depends mainly on the space gradient and is given by [6],

$\nabla_{r} D_{r}\left(\varepsilon_{e}\right) \nabla_{r} f\left(\varepsilon_{e}, r\right)=0$

Where $\varepsilon_{\mathrm{e}}$ is the total electron energy at the sheath entrance, $\mathrm{D}_{\mathrm{r}}\left(\varepsilon_{\mathrm{e}}\right)$ is the electron diffusion coefficient within the sheath and $\mathrm{f}\left(\varepsilon_{\mathrm{e}}, \mathrm{r}\right)$ is the EEDF at position $\mathrm{r}$ in the sheath probe. When $\mathrm{r} \rightarrow \infty, \mathrm{f}\left(\varepsilon_{\mathrm{e}}, \mathrm{r}\right)=\mathrm{f}_{0}\left(\varepsilon_{\mathrm{e}}\right)$ which is the undisturbed EEDF within the plasma bulk.

The electron flow $d \phi\left(\varepsilon_{e}\right)$ corresponding to electrons from energy ranging from $\varepsilon_{\mathrm{e}}$ to $\varepsilon_{\mathrm{e}}+\mathrm{d} \varepsilon_{\mathrm{e}}$, is given by [8],

$d \phi\left(\varepsilon_{e}\right)=-D_{r}\left(\varepsilon_{e}\right) \frac{\partial n_{e}\left(\varepsilon_{e}\right)}{\partial r}=-D_{r}\left(\varepsilon_{e}\right) \frac{\partial\left(f\left(\varepsilon_{e}, r\right) d \varepsilon_{e}\right)}{\partial r}$.

The change of EEDF due to the electron diffusion through the sheath can be written,

$$
\left[f_{r}\left(\varepsilon_{e}\right)-f_{0}\left(\varepsilon_{e}\right)\right] d \varepsilon_{e}=-\int_{r 0}^{r} \frac{d \phi\left(\varepsilon_{e}\right)}{D_{r}\left(\varepsilon_{e}\right)} d r=d j_{e}\left(\varepsilon_{e}\right) \int_{r 0}^{r} \frac{d r}{e D_{r}\left(\varepsilon_{e}\right) S}
$$

$\mathrm{S}$ is the collecting probe area, $\mathrm{dj}_{\mathrm{e}}\left(\varepsilon_{\mathrm{e}}\right)=-\mathrm{eSd} \phi\left(\varepsilon_{\mathrm{e}}\right)$ is the electron current due to electron energy ranging from $\varepsilon_{\mathrm{e}}$ to $\varepsilon_{\mathrm{e}}+\mathrm{d} \varepsilon_{\mathrm{e}}$.

From EQ(3), it can be seen that the larger diffusion coefficient value the lower is the change in EEDF. Using the Langmuir law, the electron current collected by a probe biased at a negative potential $\mathrm{V}$ is written in position $\mathrm{r}$ within the sheath,

$d j_{e}\left(\varepsilon_{e}\right)=g \varepsilon_{e}^{1 / 2} f_{r}\left(\varepsilon_{e}\right)\left[1-\frac{e V}{\varepsilon_{e}}\right] d \varepsilon_{e}$,

where $\mathrm{g}$ is a constant.

Using this equation and $\mathrm{EQ}(3)$, we obtain for the total electron current collected by a negatively biased probe, the equation first proposed by Swift [8],

$$
j_{e}=g \int_{e V}^{\infty} \frac{\left(\varepsilon_{e}-e V\right) \varepsilon_{e}^{-1 / 2} f_{0}\left(\varepsilon_{e}\right) d \varepsilon_{e}}{\left[1+\left(\frac{\left(\varepsilon_{e}-e V\right)}{\varepsilon_{e}}\right) \Psi\left(\varepsilon_{e}\right)\right]}
$$

With, 
$\Psi\left(\varepsilon_{e}\right)=g \varepsilon_{e}^{1 / 2} \int_{r_{p}}^{r} \frac{d r}{D_{r}\left(\varepsilon_{e}\right) S}$.

These equations show that the electron current collected by the negatively biased probe depends on the parameter $\Psi\left(\varepsilon_{\mathrm{e}}\right)$, called the electron diffusion parameter (or electron sink parameter). This parameter is function of the electron diffusion coefficient through the sheath. Arslanbekov [6] has shown that the knowledge of the distribution potential profile through the sheath is required to calculate the diffusion parameter in condition of collisional-probe space charge layer. However the determination of this parameter becomes very difficult without hypothesis on the potential profile. Different authors have attempted to model the electron diffusion within the sheath to determine an analytical equation for the diffusion parameter. However, these models need hypothesis and approximations and until now, they give erroneous results in the case of cold plasmas $[5,6,8]$.

In a previous work, we have developed a numerical method to directly determine the diffusion parameter values from experimental data without model or hypothesis [9].

In this method we use of $\mathrm{EQ}(4)$ and of its first derivative function with respect to $\mathrm{V}$,

$$
\frac{\partial j_{e}(V)}{\partial V}=g \int_{e V}^{\infty} \frac{-e \varepsilon_{e}^{-1 / 2} f_{0}\left(\varepsilon_{e}\right) d \varepsilon_{e}}{\left[1+\left(\frac{\varepsilon_{e}-e V}{\varepsilon_{e}}\right) \Psi\left(\varepsilon_{e}\right)\right]^{2}} .
$$

Then we consider the two factors $A=\frac{\partial j_{e}(V)}{\partial \varepsilon_{e}}$ and $B=\frac{\partial\left(\frac{\partial j_{e}(V)}{\partial V}\right)}{\partial \varepsilon_{e}}$. Using EQ(4) we have,

$$
A=\frac{\partial j_{e}(V)}{\partial \varepsilon_{e}}=g \frac{\left(\varepsilon_{e}-e V\right) \varepsilon_{e}^{-1 / 2} f_{0}\left(\varepsilon_{e}\right) d \varepsilon_{e}}{\left[1+\left(\frac{\left(\varepsilon_{e}-e V\right)}{\varepsilon_{e}}\right) \Psi\left(\varepsilon_{e}\right)\right]}=\frac{\partial j_{e}(V)}{\partial V} \frac{\partial V}{\partial \varepsilon_{e}}
$$

and using the first derivative function of $\mathrm{EQ}(4)$ versus $\mathrm{V}$ we have, 
$B=\frac{\partial\left(\frac{\partial j_{e}(V)}{\partial V}\right)}{\partial \varepsilon_{e}}=g \frac{-e \varepsilon_{e}^{-1 / 2} f_{0}\left(\varepsilon_{e}\right) d \varepsilon_{e}}{\left[1+\left(\frac{\varepsilon_{e}-e V}{\varepsilon_{e}}\right) \Psi\left(\varepsilon_{e}\right)\right]^{2}}=\frac{\partial^{2}\left(j_{e}(V)\right)}{\partial V^{2}} \frac{\partial V}{\partial \varepsilon_{e}}$

The diffusion parameter can be deduced from the ratio A/B,

$$
\Psi\left(\varepsilon_{e}\right)=-\left[\frac{e}{E_{e}} X+1\right] \frac{\varepsilon_{e}}{E_{e}}, \quad \quad \operatorname{EQ}(5)
$$

with

$$
X=\frac{A}{B}=\frac{\frac{\partial j_{e}(V)}{\partial V}}{\frac{\partial_{e}^{2} j_{e}(V)}{\partial V^{2}}}
$$

and

$E_{e}=\left(\varepsilon_{e}-e V\right)$

So, $\Psi\left(\varepsilon_{e}\right)$ can be calculated directly using the first and second derivative functions with respect to the applied voltage $\mathrm{V}$ of the $\mathrm{I}-\mathrm{V}$ probe characteristic. Only experimental results are used and no model is needed. It can be seen that for $E_{e}$ constant the change of $\Psi\left(\varepsilon_{\mathrm{e}}\right)$ is proportional to the product $\mathrm{VX}$ of the biased voltage with the ratio $\mathrm{X}$ of the first to the second derivative of the electron current with respect to $\mathrm{V}$.

Using this numerical method, we have calculated the value of the diffusion parameter under different experimental conditions and study the change of this value with plasma parameters.

\section{Experiments and discussion.}

Experiments have been performed by means of a cylindrical Langmuir probe (diameter $0.5 \mathrm{~mm}$, length $6 \mathrm{~mm}$ ) located in the middle of a cylindrical reactor made of OFHC copper (diameter $90 \mathrm{~mm}$, length $97 \mathrm{~mm}$ ) along the cylinder axis. Microwave plasma working at $2.45 \mathrm{GHz}$ with powers ranging from $1500 \mathrm{~W}$ to $1800 \mathrm{~W}$ is maintained in hydrogen at $0.19 \mathrm{~Pa}$. A magnetic field intensity ranging from $80 \mathrm{mT}$ to $120 \mathrm{mT}$ is applied by means of four coils. It is parallel to the reactor axis and to the probe collector. More details of the experimental set up and a scope of the reactor are given in [10]. The ion current collected by the probe at saturation is determined at large negative biased voltage ranging from $-150 \mathrm{~V}$ to $-100 \mathrm{~V}$, when all electrons are repelled. The electron current is calculated by substracting the ion current at 
saturation to the measured total current. Experiments have been performed in different positions along the reactor axis. The magnetic field is determined by means of a Hall probe with error bellow $2 \%$. Figure 1 shows the magnetic field profile along the reactor axis with a simplified view of the reactor in the inset. The plasma chamber is located between $\mathrm{z}=0 \mathrm{~mm}$ to $\mathrm{z}=97 \mathrm{~mm}$.

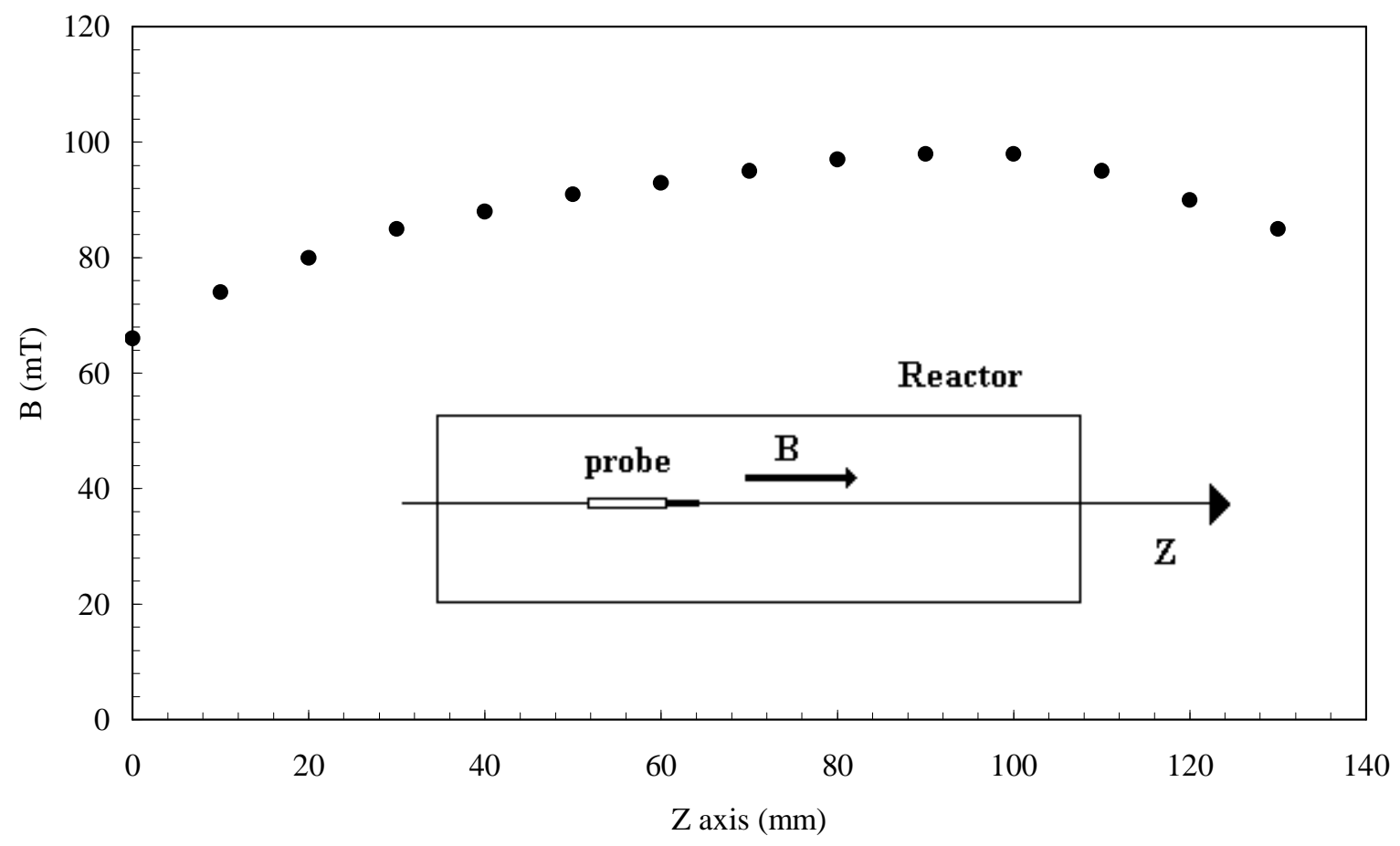

Figure1. The magnetic field profile along the z axis and a simplified set up of the reactor.

Figure 2 shows the evolution of the diffusion parameter value versus $E_{e}=\varepsilon_{e}-e V$. It is calculated using $E Q(5)$ for different applied voltage $V_{\text {app }}$, which correspond to a bias voltage $V=V_{P}-V_{\text {app }}$, $\mathrm{V}_{\mathrm{P}}$ is the plasma potential equal to $42,08 \mathrm{~V}$. The derivative functions of $\mathrm{je}(\mathrm{V})$ versus $\mathrm{V}$ are obtained using the numerical differentiation method for noisy signal proposed in ref $[11,12]$. Measurements have been performed at $43 \mathrm{~mm}$ from the wave launcher exit on the z-axis, using a microwave power equal to $1500 \mathrm{~W}$. It can be seen that the diffusion parameter depends on the biased voltage and is strongly decreasing with increasing electron energy.

Whatever the applied bias voltage, the diffusion parameter value versus electron energy $\varepsilon_{\mathrm{e}}$ can be fitted using a function $\Psi\left(\varepsilon_{e}\right)=A \varepsilon_{e}^{-\alpha}$, with $\alpha$ ranging from 0.8 to 1.4 and " $\mathrm{A}$ " ranging from 80 to 500 for an hydrogen pressure of $0.2 \mathrm{~Pa}$, a power ranging from $1500 \mathrm{~W}$ to $1800 \mathrm{~W}$ and a 
magnetic field intensity ranging from $70 \mathrm{mT}$ to $100 \mathrm{mT}$. A typical fit of experimental diffusion parameter values using this function is shown on Figure 3.

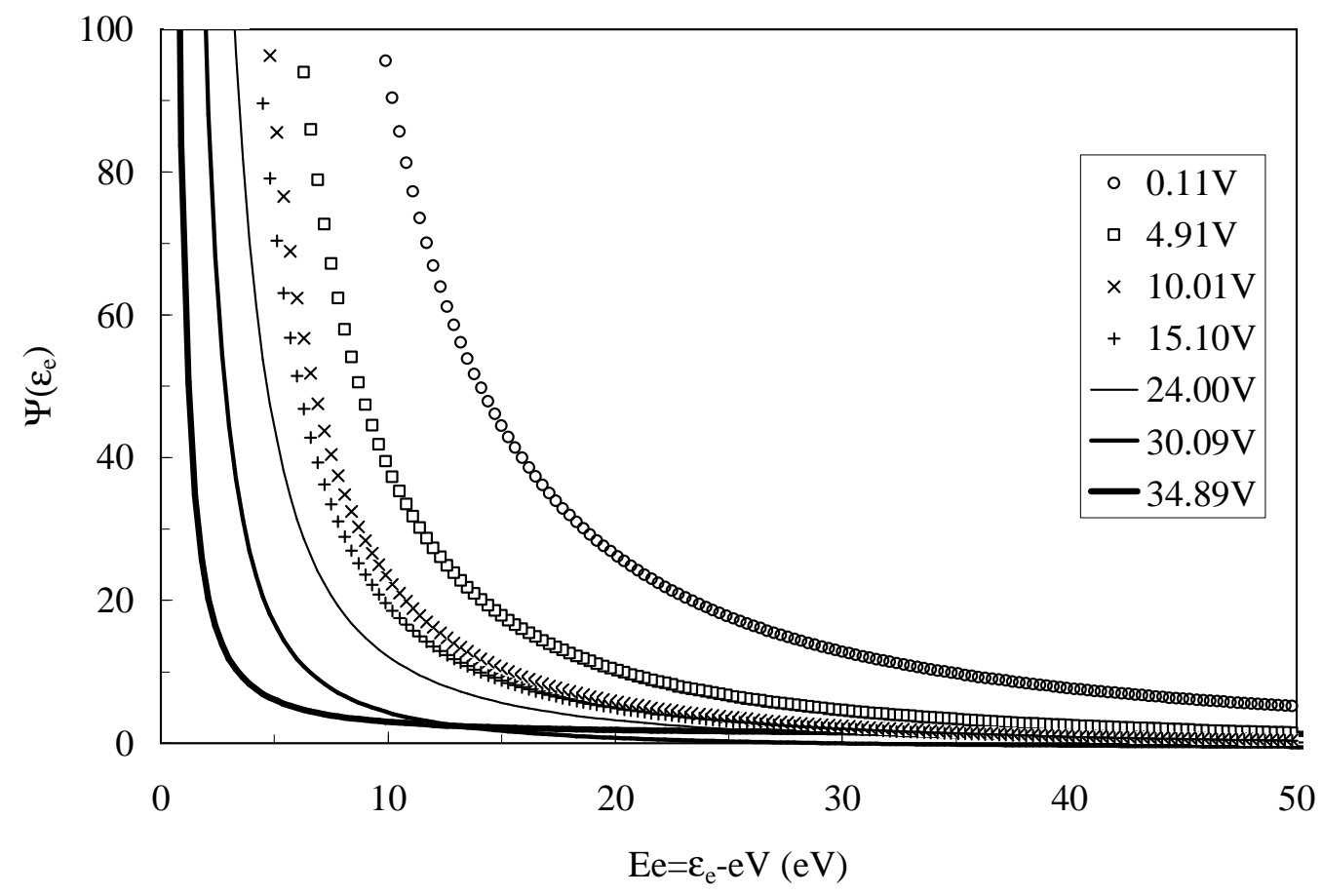

Figure2. $\Psi\left(\varepsilon_{e}\right)$ versus $E e=\varepsilon_{e}-e V$, in the case of different biased voltages.

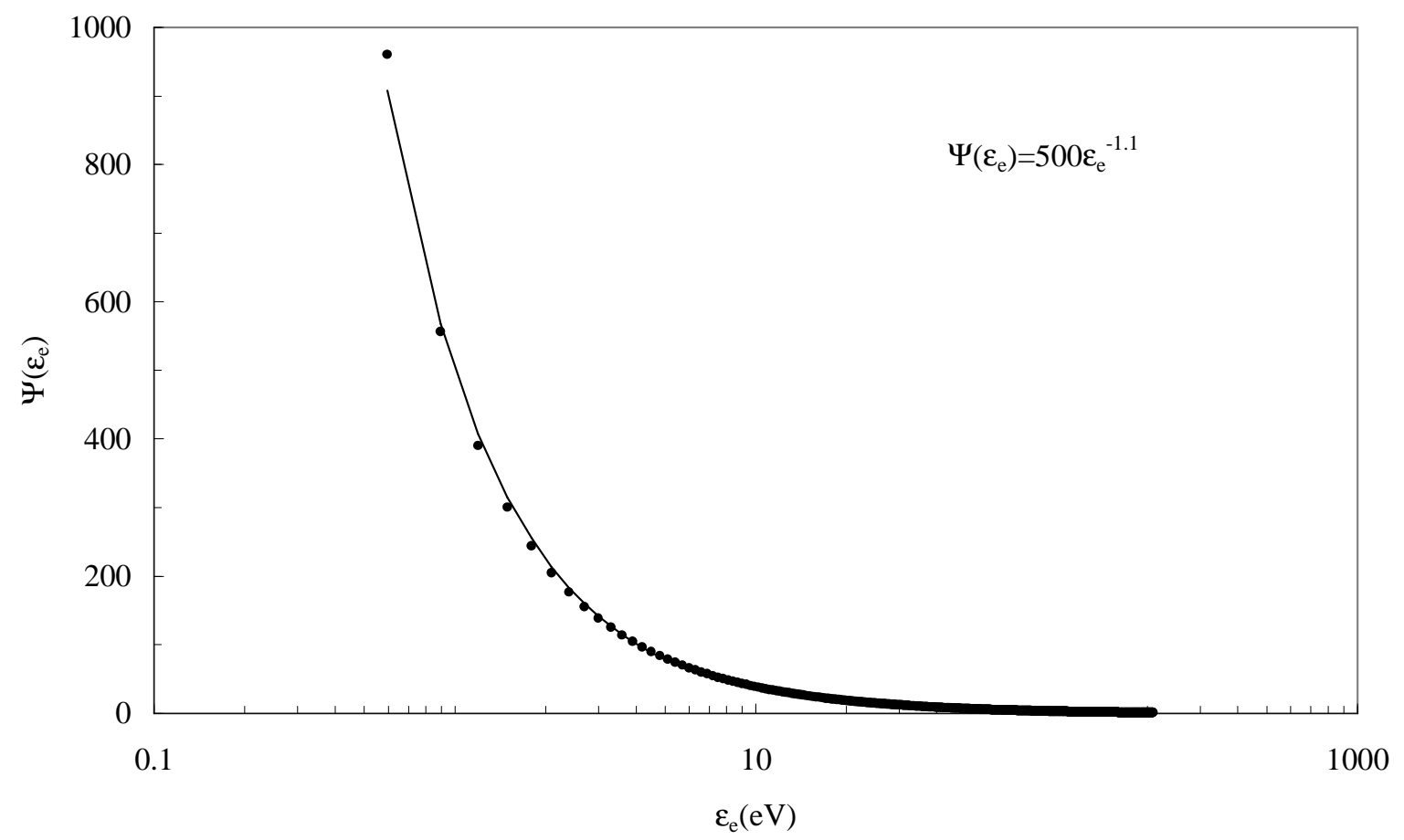


Figure3. Fit of the diffusion parameter versus electron energy, in the case of hydrogen pressure of 0.2Pa, power of $1800 \mathrm{~W}$ and magnetic field of $85 \mathrm{mT}$. Measurements are performed along the reactor axis at a biased voltage of $0,1 \mathrm{~V}$.

Using EQ(5) and comparing the diffusion parameter corresponding to two different values of biased voltage $\mathrm{V}_{1}$ and $\mathrm{V}_{0}$, we have,

$\frac{\Psi_{V 1}\left(E_{e}\right)}{\Psi_{V 0}\left(E_{e}\right)}=\frac{\left[\left(\frac{e}{E_{e}}\right) X_{1}+1\right]}{\left[\left(\frac{e}{E_{e}}\right) X_{0}+1\right]} \frac{\left(E_{e}+e V_{1}\right)}{\left(E_{e}+e V_{0}\right)}$

Figure4 shows the change of $\Psi_{\mathrm{V} 1}\left(\mathrm{E}_{\mathrm{e}}\right) / \Psi_{\mathrm{V} 0}\left(\mathrm{E}_{\mathrm{e}}\right), \mathrm{X}_{1} / \mathrm{X}_{0}, \mathrm{dI} / \mathrm{dV}$ and $\mathrm{d}^{2} \mathrm{I} / \mathrm{dV}^{2}$ versus biased voltage $\mathrm{V}_{\mathrm{p}}-\mathrm{V}$, corresponding to measurements performed at $43 \mathrm{~mm}$ on the z-axis from the wave launcher exit and using a microwave power equal to $1500 \mathrm{~W}$. Calculations of $\Psi_{\mathrm{V} 1}\left(\mathrm{E}_{\mathrm{e}}\right) / \Psi_{\mathrm{V} 0}\left(\mathrm{E}_{\mathrm{e}}\right)$ have been performed at $\mathrm{E}_{\mathrm{e}}=10^{-5} \mathrm{eV}$. This figure shows the strong dependence of $\Psi_{\mathrm{V}}\left(\mathrm{E}_{\mathrm{e}}\right)$ with $\mathrm{X}$.

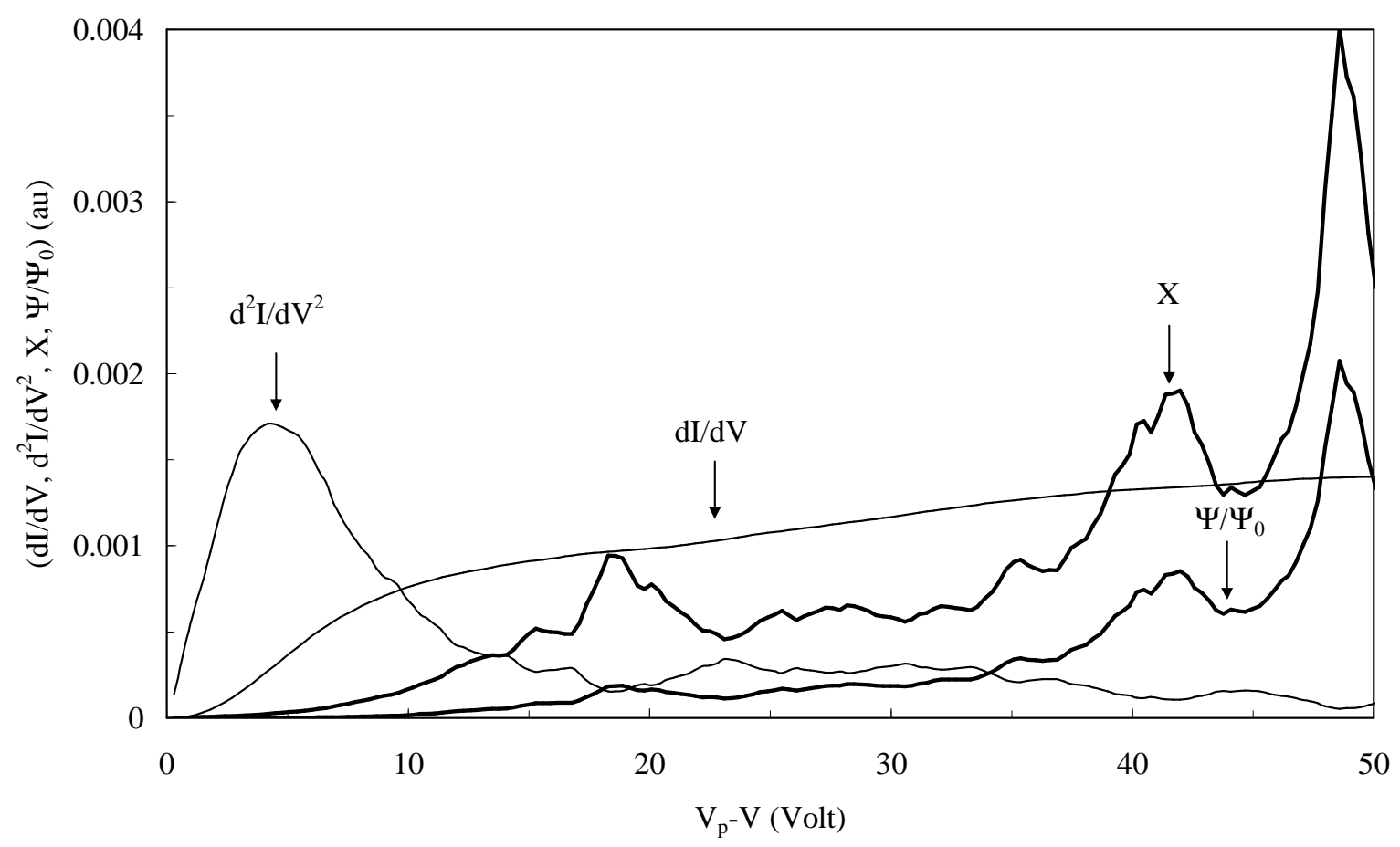


Figure4. Comparison of the first and second derivative of the I-V probe characteristic and of $X$ and $\Psi / \Psi_{0}$, versus $V_{p}-V$; Measurements are performed at $1500 \mathrm{~W}, 0.2 \mathrm{~Pa} \mathrm{H}_{2}$ and at $43 \mathrm{~mm}$ of the wave launcher exit on the Z-axis in the middle of the reactor and for $E_{e}=10^{-5} \mathrm{eV}$.

According to Dote [13], in the case of a cylindrical probe parallel to the magnetic field and when $\omega^{2} \tau^{2}>>1$, the ratio $\frac{I_{e}\left(\varepsilon_{e}\right)}{I_{e 0}\left(\varepsilon_{e}\right)}$ of the electron current collected with magnetic field on the electron current collected without magnetic field, is given by,

$$
\frac{I_{e}\left(\varepsilon_{e}\right)}{I_{e 0}\left(\varepsilon_{e}\right)}=\left[1+\frac{r_{p}}{\lambda_{e}} \log \left(\frac{r_{p}+d}{r_{p}}\right)\left(1+\omega^{2} \tau^{2}\right)\right]^{-1}=\left[1+A \omega^{2} \tau^{2}\right]^{-1}
$$

Where, $\mathrm{A}$ is a constant, $\omega$ and $\tau=\lambda_{\mathrm{e}} / \mathrm{v}_{\mathrm{e}}$ are the angular frequency and the time between two electron-neutral collisions, respectively. $\lambda_{\mathrm{e}}$ and $\mathrm{v}_{\mathrm{e}}$ are the electron mean free path and the electron velocity at the probe sheath entrance. It is worth noting that in $\mathrm{EQ}(7)$, Dote considers a monokinetic electron current of energy $\varepsilon_{\mathrm{e}}$.

Considering the Langmuir law for the electron current $\mathrm{I}_{\mathrm{e} 0}$ collected when no magnetic field is applied and considering EQ(4) for the electron current $I_{e}$ collected when a magnetic field is applied. The ratio $\frac{I_{e}\left(\varepsilon_{e}\right)}{I_{e 0}\left(\varepsilon_{e}\right)}$ can be written in the case of a mono kinetic electron current,

$$
\frac{I_{e}\left(\varepsilon_{e}\right)}{I_{e 0}\left(\varepsilon_{e}\right)}=\frac{4}{3 \gamma}\left[1+\frac{\left(\varepsilon_{e}-e V\right)}{\varepsilon_{e}} \Psi\left(\varepsilon_{e}\right)\right]^{-1}
$$

The comparison with EQ(7) gives,

$$
\frac{4}{3 \gamma}\left[1+\frac{\left(\varepsilon_{e}-e V\right)}{\varepsilon_{e}} \Psi\left(\varepsilon_{e}\right)\right]^{-1}=\left[1+A \omega^{2} \tau^{2}\right]^{-1}
$$

This equation shows that $\Psi\left(\varepsilon_{\mathrm{e}}\right)$ is proportional to $\omega^{2} \tau^{2}$ and consequently to $\mathrm{B}^{2} \varepsilon_{\mathrm{e}}{ }^{-1}$, because $\omega=\frac{e B}{m_{e}}$ and $\tau=\frac{\lambda_{e}}{v_{e}}=\lambda_{e} \sqrt{\frac{m_{e}}{2 \varepsilon_{e}}}$.

This confirms the previous experimental results obtained using EQ(5) and shown in Figure2 and Figure3. The electron diffusion parameter depends on $\varepsilon_{\mathrm{e}}{ }^{-1}$. 
Based on the work of Mal'kov [4], who has calculated the electron current under the conditions of a collisional near-probe space-charge layer, authors [5,6] obtained $\Psi\left(\varepsilon_{\mathrm{e}}\right)$ proportional to $\omega \tau$, i.e. to $\mathrm{B} \varepsilon_{\mathrm{e}}{ }^{-1 / 2}$. These results disagree with our results, with Swift model [8] and with Dote et al theory [13]. Based on their models these authors [4,5,6] overestimate $\Psi\left(\varepsilon_{e}\right)$ by a factor 100 , in such cold plasma. [9]

As previously shown,

$$
\Psi\left(\varepsilon_{e}\right)=g \varepsilon_{e}^{1 / 2} \int_{r p}^{\infty} \frac{d r}{D_{r} e S}
$$

Where $D_{r}$ is the normal component of the electron diffusion coefficient, which in a magnetic field parallel to the probe is equal to $D_{r}=\frac{D}{\left(1+\omega^{2} \tau^{2}\right)}$, where $\mathrm{D}$ is the electron diffusion coefficient without magnetic field. $D=\lambda_{e}^{2} v, v$ is the electron collision frequency [14].

The electron diffusion parameter depends on the sheath thickness, on the potential profile through the sheath, on the diffusion coefficient and consequently on the Larmor radius and the electron energy incoming in the probe sheath. So, it depends on plasma parameters as the electron mean energy and density, $\left\langle\varepsilon_{\mathrm{e}}\right\rangle$ and $\mathrm{n}_{\mathrm{e}}$, respectively and on the magnetic field intensity B.

We assume in a first approximation a constant diffusion coefficient through the sheath. This assumption has been used in previous work to determine $\Psi\left(\varepsilon_{e}\right) / 5,6 /$. So, we have,

$$
\Psi\left(\varepsilon_{e}\right)=g^{\prime} \int_{r p}^{\infty} \frac{\left(1+\omega^{2} \tau^{2}\right) d r}{\lambda_{e} e S}=g^{\prime} \int_{r p}^{\infty} \frac{\omega^{2} \tau^{2} d r}{\lambda_{e} e S}, \text { if } \omega^{2} \tau^{2}>>1
$$

In the case of a cylindrical probe with length $\mathrm{L}$ and radius $\mathrm{r}_{\mathrm{p}}, \Psi\left(\varepsilon_{e}\right)$ can be written,

$$
\Psi\left(\varepsilon_{e}\right)=\frac{g^{\prime}}{2 \pi L e} \int_{r p}^{\infty} \frac{\omega^{2} \tau^{2} d r}{\lambda_{e} r}=g^{\prime \prime} B^{2} \lambda_{e} \varepsilon_{e}^{-1} \log \left(\frac{x_{d}}{r_{p}}\right)
$$

Where $\mathrm{x}_{\mathrm{d}}$ is the sheath thickness formed around the probe. Assuming in a first approximation that $x_{d}=\alpha \lambda_{D}$ where $\lambda_{\mathrm{D}}$ is the Debye lengths, with $\lambda_{D}=\sqrt{\left(\frac{2 / 3\left\langle\varepsilon_{e}\right\rangle \varepsilon_{0}}{n_{e} e^{2}}\right)}$, where $\left\langle\varepsilon_{\mathrm{e}}>\right.$ and $\mathrm{n}_{\mathrm{e}}$ are the electron mean energy and density, respectively. Under these conditions and if $x_{d}<<r_{p}$, $\Psi\left(\varepsilon_{\mathrm{e}}\right)$ should be proportional to $\mathrm{B}^{2} \lambda_{\mathrm{e}} \varepsilon_{\mathrm{e}}{ }^{-1} \lambda_{\mathrm{D}}$.

This model $(\mathrm{EQ}(8))$ has been applied to experimental data obtained within four series a, b, $c$ and $\mathrm{d}$. The incident power and the pressure are equal to $1500 \mathrm{~W}$ and $0.19 \mathrm{~Pa}$, respectively for the series $\mathrm{a}, \mathrm{b}$ and $\mathrm{c}, 1800 \mathrm{~W}$ and $0.2 \mathrm{~Pa}$, respectively for the series $\mathrm{d}$. In the case of the series 
$\mathrm{b}$, measurements are performed in a region corresponding to electron cyclotron resonance (ECR) conditions. Figure 5 shows the change of $\sqrt{\frac{\Psi\left(\varepsilon_{e}=1 e V\right)}{\lambda_{D}}}$ versus $\mathrm{B}$ in the case of the four series. The electron density and mean energy value needed to calculate $\lambda_{d}$ have been calculated integrating the measured EEDF using the method described in [9].

The correlation factor of the linear regression with experimental data remains low for most of the series and the slope of the linear regression changes according to the series i.e. with the experimental conditions (pressure, microwave incident power, position and magnetic field intensity). The results show that the ratio $\sqrt{\frac{\Psi\left(\varepsilon_{e}=1 e V\right)}{\lambda_{D}}}$ (i.e. $\sqrt{\frac{A}{\lambda_{D}}}$ when the function is fitted with $\Psi\left(\varepsilon_{e}\right)=A \varepsilon_{e}^{-\alpha}$, as shown on Figure 3) slightly increases with increasing magnetic field intensity. However, this simple model is inefficient to describe the phenomena because of the low correlation between theory and experiment. This can be ascribed to the rough assumptions concerning the sheath thickness and the diffusion coefficient but also to the strong dispersion in experimental values depending on plasma conditions. The change in plasma parameters leads to change in electron motion, sheath thickness or potential profile within the sheath and consequently in the values of the electron diffusion parameters. Figure 6,7 and 8 show the values of plasma parameters along the $\mathrm{z}$ axis of the reactor and between the different series. In the sake of clarity we use "spline interpolation" between the points of the different series. The values of the electron density and mean energy are calculated integrating the EEDF which is determined using the method described in ref [9]. The plasma and the floating potential are determined at the inflection point of the I-V curve and at the crossing point with the $\mathrm{x}$ axis, respectively.

It can be seen that the electron density and the plasma and floating potentials increase with increasing B. However, the mean electron energy decreases with increasing B, probably because of the decrease of the Larmor radius and the increase of the electron neutral collision frequency with increasing B. 


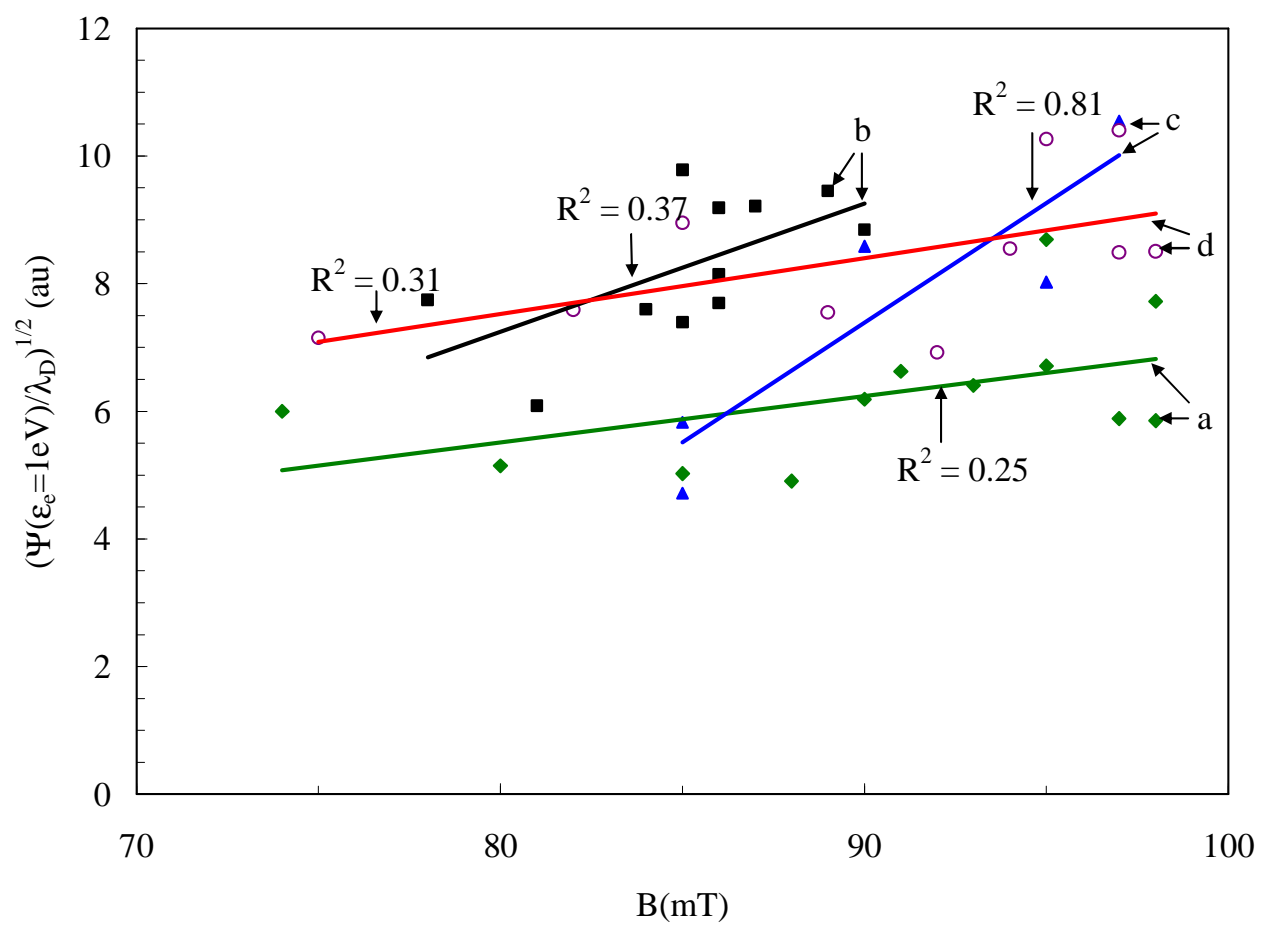

Figure5. Change of $\sqrt{\frac{\Psi\left(\varepsilon_{e}=1 \mathrm{eV}\right)}{\lambda_{D}}}$ versus $B$ in the case of the series $a, b, c, d$, at a biased voltage $V=0.1 V$. Linear regressions are added on the graph.

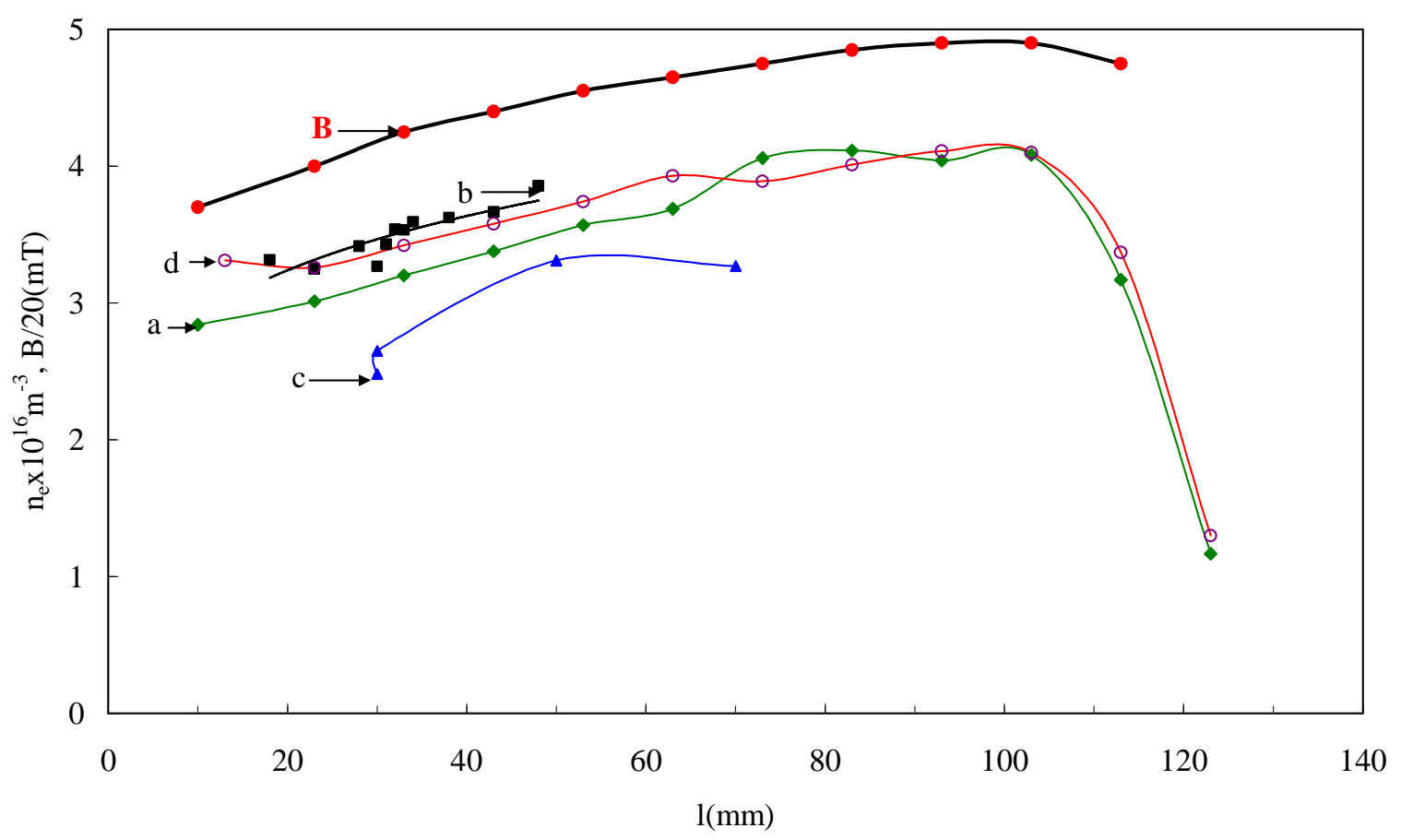

Figure6. Electron density and magnetic field intensity versus the position along the longitudinal reactor axis, in the case of the four previous series $a, b, c, d$ used in Figure5. 


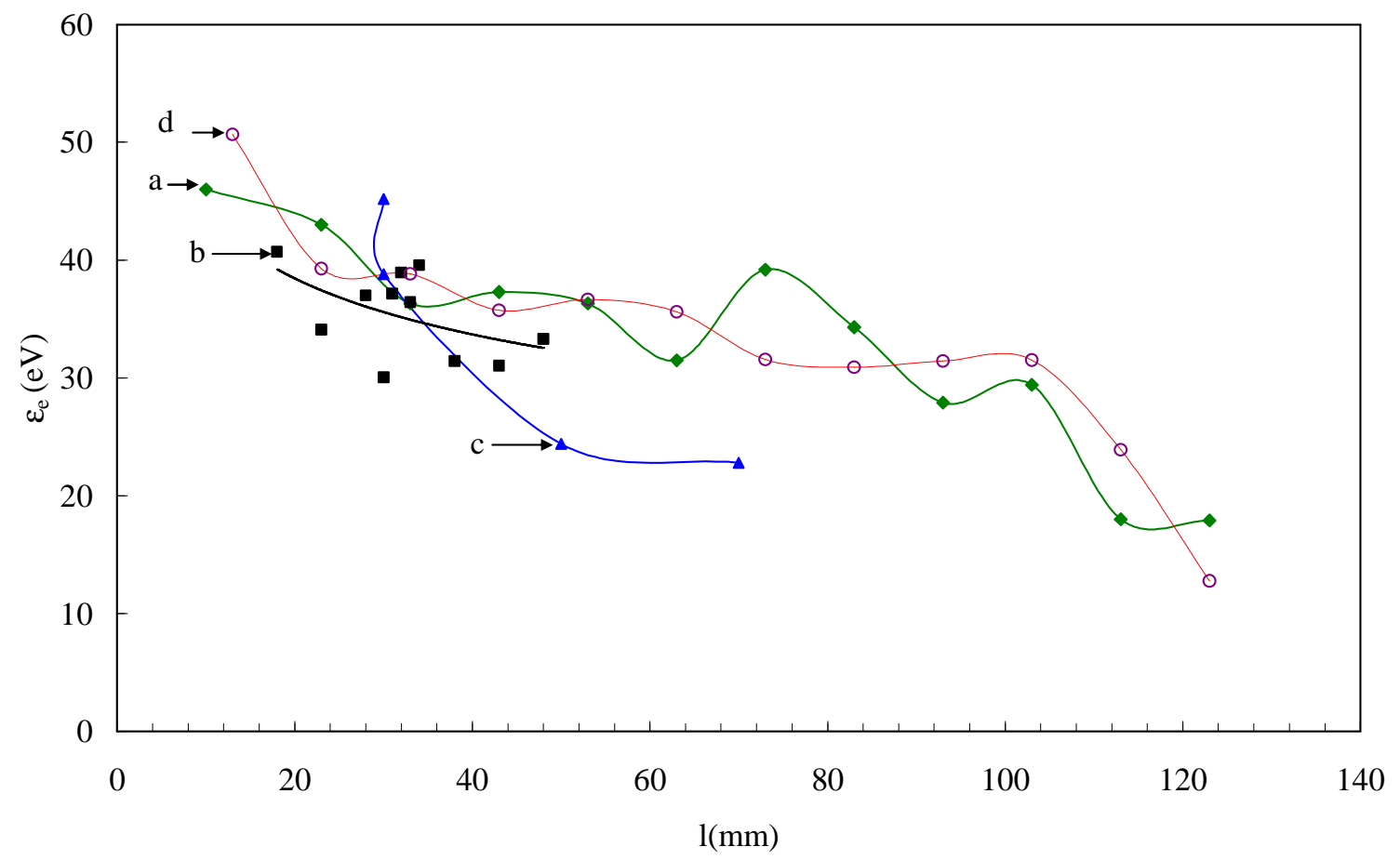

Figure7. Mean electron energy versus the position along the longitudinal reactor axis, in the case of the four previous series $a, b, c, d$ used in Figure5.

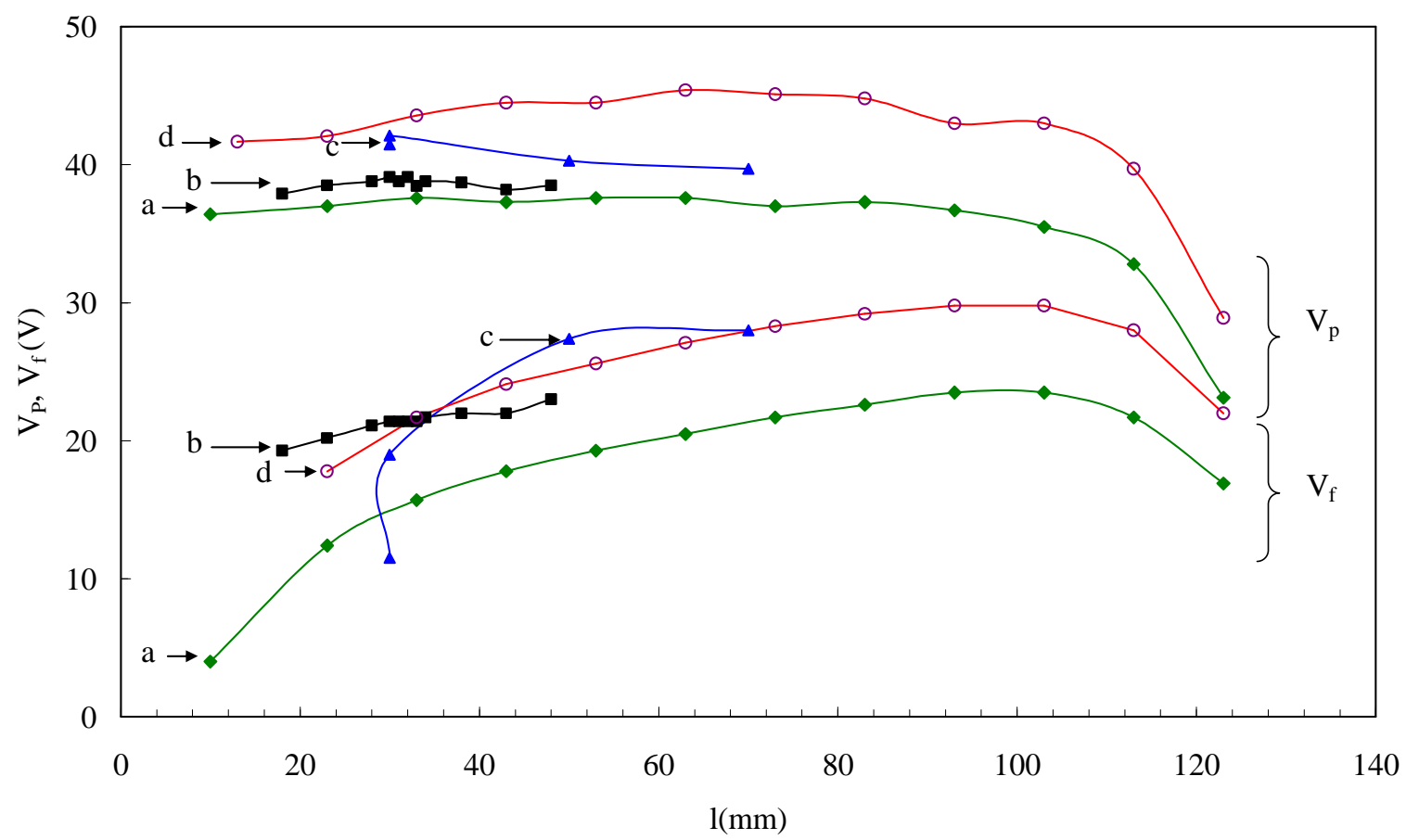

Figure8. Plasma $\left(V_{p}\right)$ and floating $\left(V_{f}\right)$ potentials versus the position along the longitudinal reactor axis, in the case of the four previous series $a, b, c, d$ used in Figure5. 
The phenomena produced under such experimental conditions cannot be correlated by a simple model. So another attempt to correlate the electron diffusion parameter to the magnetic field is given as follows.

The diffusion parameter has been previously shown to be fitted by the function $\Psi\left(\varepsilon_{e}\right)=A \varepsilon_{e}^{-\alpha}$. Using diffusion parameter values calculated with the experimental method for series a, b, c and $\mathrm{d}$, we have determined an equation $A=f\left(V, n_{e},\left\langle\varepsilon_{e}\right\rangle, B\right)$. In this equation, $\mathrm{V}, \mathrm{n}_{\mathrm{e}},\left\langle\varepsilon_{\mathrm{e}}\right\rangle$ and $\mathrm{B}$ are the biased voltage of the probe, the electron density, the mean energy and the magnetic field intensity, respectively. Figure 9 shows the change of $\left(n_{e}^{x}\left\langle\varepsilon_{e}\right\rangle^{y} \log (A)\right)$ versus magnetic field intensity $\mathrm{B}$, when $\mathrm{V}=0.1 \mathrm{~V}$. The best agreement with a linear regression is obtained for $\mathrm{x}=4.3$ and $\mathrm{y}=-1.67$ and it corresponds to a correlation factor equal to 0.75 . The exponent of density and mean energy are arbitrary values which cannot be justified without a better knowledge of the phenomena. This linear regression gives information concerning the change of the magnetic field intensity within the plasma.

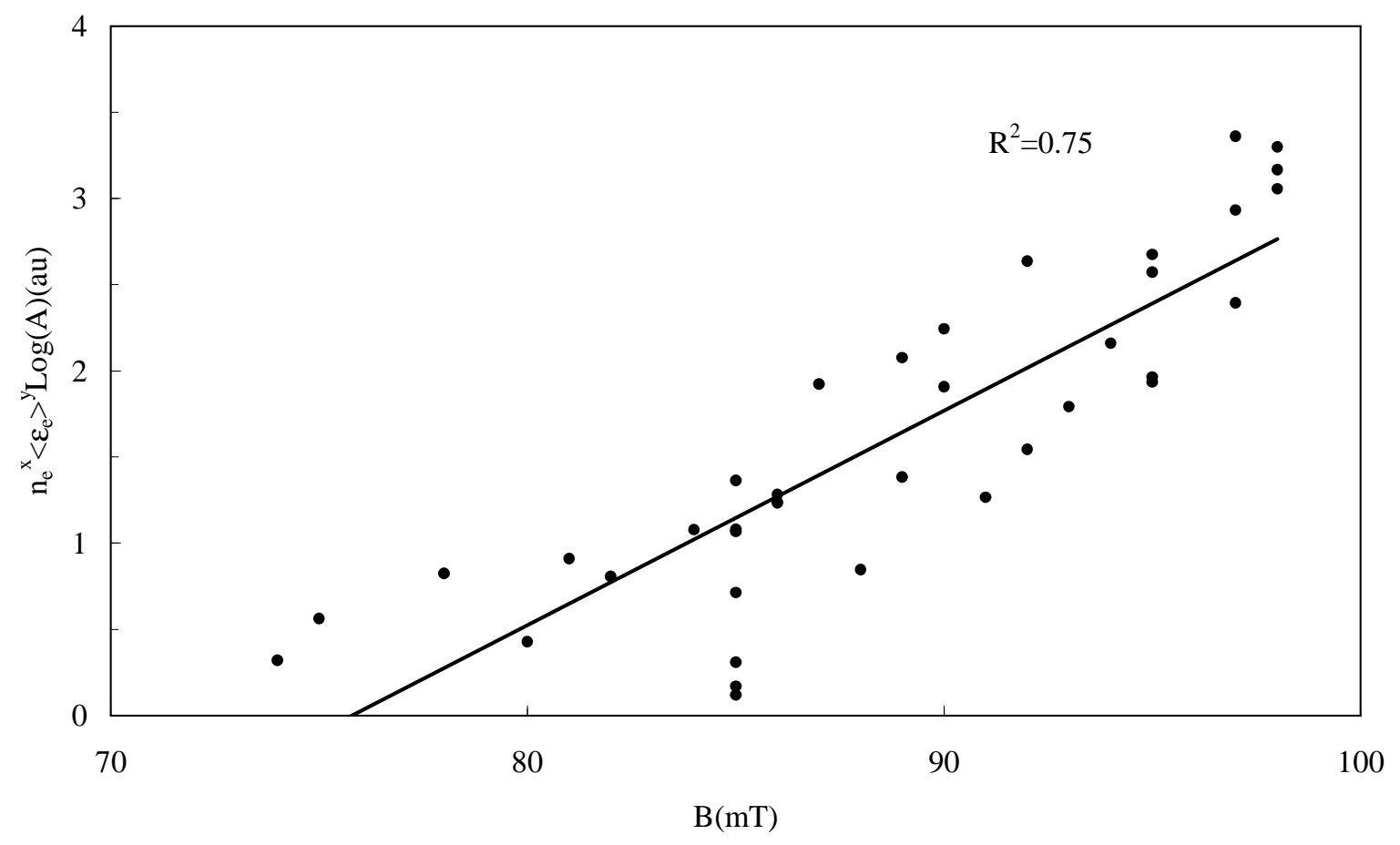

Figure9. Linear regression of $\left(n_{e}^{x}\left\langle\varepsilon_{e}\right\rangle^{y} \log (A)\right)$ versus $B$, at a biased voltage $V=0.1 \mathrm{~V}$.

\section{Conclusion.}

This work is devoted to the study of the electron diffusion parameter in magnetized plasma. Experiments are performed in a cylindrical reactor, in microwave magnetized plasma working at $2,45 \mathrm{GHz}$. We have shown that the diffusion parameter depends strongly on the ratio of the 
first to the second derivative function versus $\mathrm{V}$ of the probe characteristic I-V. The diffusion parameter can be fitted using the general law $\Psi\left(\varepsilon_{e}\right)=A \varepsilon^{-n}$ with n ranging from 0.8 to 1.4 and A ranging from 80 to 500 according to the experimental condition at $0.19 \mathrm{~Pa}$ and for a microwave power ranging from $1500 \mathrm{~W}$ to $1800 \mathrm{~W}$ and a magnetic field intensity ranging from $80 \mathrm{mT}$ to $120 \mathrm{mT}$. These results agree with Dote and Swift model $[7,10]$ and show that the diffusion parameter is proportional to $\varepsilon_{\mathrm{e}}{ }^{-1}$. It disagrees with Mal'kov, Arslanbekov or Popov model [4-6] model which obtain a diffusion parameter proportional to $\varepsilon_{\mathrm{e}}^{-1 / 2}$. Until now none model is really efficient to calculate the diffusion parameter, probably because of the real potential profile and the electron motion within the sheath formed around the probe collector are unknown or because of a wrong estimation of the sheath thickness. According to Rozhansky [7] in presence of magnetic field, the anomalous diffusion can have an effect on the charged particles density profile near the probe and there can be a significant expansion of the sheath.

However, we have obtained a linear regression for $\left(n_{e}^{x}\left\langle\varepsilon_{e}\right\rangle^{y} \log (A)\right)$ versus B at a biased voltage $\mathrm{V}=0.1 \mathrm{~V}$. The best agreement with experimental data is obtained for $\mathrm{x}=4,3$ and $\mathrm{y}=-$ 1,67 and corresponds to a correlation factor equal to 0.75 . Such linear regression can be used to measure the magnetic field intensity within the reactor, with a more or less large error according to the correlation factor.

\section{Refrerences.}

[1]. N. Hershkowitz, in «Plasma diagnostics » vol 1, Editors O. Auciello and D.L. Flamm (Academic press, N.Y) 113-183 (1989).

[2] F.F. Chen, electric probes in "plasma Diagnostic Techniques", Editors R.H. Huulestone and S.L. Leonard (Academic Press, N.Y) 113-200 (1965).

[3]. Physics of Radio-Frequency Plasmas P. Chabert, N. Braithwaite, Ed Cambridge University Press, Cambridge. p 294-295 (2011).

[4] M.A. Mal'kov, High Temp. 29429 614-619 (1991).

[5]. T.K. Popov, P. Ivanova, M. Dimitrova, J. Kovačič, T. Gyergyek, M. Čerček, Plasma Sources Sci. Technol. 21025004 (2012)(10pp).

[6]. R.R. Arslanbekov, N.A. Khromov, A. Kudryavtsev, Plasma Source Sci. Technol. 3 528538 (1994).

[7]. V.A. Rozhansky, A.A. Ushakov, S.P. Voskoboynikov, Nucl. Fusion. 39613 (1999). 
[8]. J.D. Swift, Proc. Phys. Soc. 79 697-701 (1962).

[9]. J.L. Jauberteau, I. Jauberteau, O. D. Cortázar, A. Megía-Macías, Contrib. Plasma Phys. 602 (2019) (9pp).

[10]. A Megía-Macías, O D Cortázar, A Vizcaíno-de-Julián, Rev. Sci. Instrum. 85033310 (2014) (11pp). doi: 10.1063/1.4869343

[11]. J.L. Jauberteau, I. Jauberteau, Meas. Sci. Technol. 18 1235-49 (2007).

[12]. F. Jauberteau, J.L. Jauberteau, Applied Mathematics and Computation 215 2283-2297 (2009).

[13]. T. Dote, H. Amemiya, T. Ichimiya, Jpn. J. Appl. Phys. 3, 12, 789-796 (1964).

[14]. I.P. Shkarofsky, T.W. Johnston, M.P. Bachynski, the particle kinetics of plasmas, Addison-Wesley publishing company, inc , London (1966). 